\title{
Characterization of an Asymptomatic Cohort of Severe Acute Respiratory Syndrome Coronavirus 2 (SARS- CoV-2) Infected Individuals Outside of Wuhan, China
}

\begin{abstract}
Yubo Wang, ${ }^{1, a}$ Yong He, ${ }^{1, b \odot}$ Jin Tong, ${ }^{2, a}$ Yalan Qin, ${ }^{3}$ Ting Xie, ${ }^{4}$ Jianghua Li, ${ }^{1}$ Jianrong Li, ${ }^{5}$ Jianhua Xiang, ${ }^{6}$ Yong Cui, ${ }^{7}$ Elizabeth S. Higgs, ${ }^{8}$ and Jianglin Xiang ${ }^{4, b}$
${ }^{1}$ Department of Respiratory Medicine, Daping Hospital, Army Medical University, Chongqing, China, ${ }^{2}$ Department of Respiratory Medicine, Second Affiliated Hospital of Chongqing Medical University, Chongqing, China, ${ }^{3}$ Department of Critical Care Medicine, Second Affiliated Hospital of Chongqing Medical University, Chongqing, China, ${ }^{4}$ Department of Infection, Chongqing Three Gorges Central Hospital, Chongqing, China, ${ }^{5}$ Department of Respiratory Medicine, Wanzhou District Hospital, Chongqing, China, ${ }^{6}$ Department of Respiratory Medicine, Chongqing Three Gorges Central Hospital, Chongqing, China, ${ }^{7}$ Department of Critical Care Medicine, Chongqing Three Gorges Central Hospital, Chongqing, China, and ${ }^{8}$ Division of Clinical Research, National Institute of Allergy and Infectious Diseases, National Institutes of Health, Bethesda, Maryland, USA
\end{abstract}

Purpose. We aimed to further clarify the epidemiological and clinical characteristics of asymptomatic severe acute respiratory syndrome coronavirus 2 (SARS-CoV-2) infections.

Methods. We identified close contacts of confirmed coronavirus disease 2019 (COVID-19) cases in northeast Chongqing, China, who were confirmed by real-time reverse transcription polymerase chain reaction-positive (RT-PCR+). We stratified this cohort by normal vs abnormal findings on chest computed tomography $(\mathrm{CT})$ and compared the strata regarding comorbidities, demographics, laboratory findings, viral transmission and other factors.

Results. Between January 2020 and March 2020, we identified and hospitalized 279 RT-PCR+ contacts of COVID-19 patients. 63 (23\%) remained asymptomatic until discharge; 29 had abnormal and 34 had normal chest CT findings. The mean cohort age was 39.3 years, and $87.3 \%$ had no comorbidities. Mean time to diagnosis after close contact with a COVID-19 index patient was 16.0 days, and it was 13.4 days and 18.7 days for those with abnormal and normal CT findings, respectively $(P<.05)$. Nine patients $(14.3 \%)$ transmitted the virus to others; 4 and 5 were in the abnormal and normal CT strata, respectively. The median length of time for nucleic acid to turn negative was 13 days compared with 10.4 days in those with normal chest CT scans $(P<.05)$.

Conclusions. A portion of asymptomatic individuals were capable of transmitting the virus to others. Given the frequency and potential infectiousness of asymptomatic infections, testing of traced contacts is essential. Studies of the impact of treatment of asymptomatic RT-PCR+ individuals on disease progression and transmission should be undertaken.

Keywords. COVID-19; SARS-CoV-2; asymptomatic infection; characterization.

In December 2019 a novel coronavirus, later named severe acute respiratory syndrome coronavirus 2 (SARS-CoV-2), caused a large outbreak of infectious disease, designated coronavirus disease 2019 (COVID-19). It posed a great threat to public health worldwide [1]. Compared with SARS-CoV or Middle East respiratory syndrome coronavirus, it appears to be transmitted more easily [2-4]. SARS$\mathrm{CoV}-2$ can be transmitted through a variety of modes, including droplets, close contact, aerosols, and potentially fecal-oral transmission $[5,6]$. People of all ages are generally susceptible to the virus.

Received 17 March 2020; editorial decision 19 May 2020; accepted 20 May 2020; published online May 22, 2020.

${ }^{\mathrm{a} Y}$. W. and J. T. contributed equally to this work.

${ }^{b} Y$. H. and J. X. contributed equally to this work.

Correspondence: Y. He, Department of Respiratory Medicine, 10\# Changjiang Zhi Road, Chongqing 400042, China (heyong8998@126.com).

Clinical Infectious Diseases ${ }^{\circledR} \quad$ 2020;71(16):2132-8

(C) The Author(s) 2020. Published by Oxford University Press for the Infectious Diseases Society of America. This is an Open Access article distributed under the terms of the Creative Commons Attribution-NonCommercial-NoDerivs licence (http://creativecommons.org/licenses/ by-nc-nd/4.0/), which permits non-commercial reproduction and distribution of the work, in any medium, provided the original work is not altered or transformed in any way, and that the work is properly cited. For commercial re-use, please contact journals.permissions@oup.com DOl: $10.1093 /$ cid/ciaa629
Symptomatic COVID-19 patients and asymptomatic cases are both a source of infection, and patients in the incubation period can transmit SARS-CoV-2 to other persons [7-10].

Patients with symptoms are more likely to be detected. Among patients with SARS-CoV-2, fever has been the most common symptom, followed by dry cough, dyspnea, myalgia, headache, and diarrhea [11-13]. In severe COVID-19 cases, symptoms can progress to acute respiratory distress syndrome, septic shock, and metabolic acidosis [14]. The clinical presentation of COVID-19, therefore, ranges from asymptomatic to severe respiratory illness [15]. The presentation of symptoms provides an opportunity for case detection and isolation, facilitating the interruption of transmission. Because most SARS-CoV patients had severe symptoms, they were relatively simple to identify and isolate, thus interrupting the chain of transmission. Unfortunately, COVID-19 has an incubation period from infection to onset of symptoms that lasts an average of 10 days (with a reported range of $2-14$ days) [11, 16, 17]. The virus is also able to spread from one person to another before any symptom onset [14]. It is reported that about $1.2 \%$ of 
patients have no actual clinical manifestations during the entire course of the disease [18]. Asymptomatic cases, however, particularly those with no history of contact with a known SARS-CoV-2 patient, are hard to identify. There are several case reports of SARS-CoV-2 transmission from asymptomatic individuals $[8,9,13,19]$, including a report of presumed transmission by asymptomatic carriers with normal chest computed tomography (CT) scans [20]. Together, these traits make control of SARS-CoV-2 very difficult.

The exact profile of these asymptomatic cases and their role within the wider epidemic are unclear. It is important to understand the proportion of asymptomatic or mildly ill cases and their role in driving the epidemic [21]. Since many SARS-CoV-2 infections are asymptomatic, subclinical, or very mild, the hallmarks and course of asymptomatic infection warrant further investigation [22-24]. Our goal was to document the characteristics of asymptomatic infections and identify factors associated with asymptomatic infection, enabling the formulation of corresponding strategies and control measures.

\section{METHODS}

\section{Data Sources}

We conducted a retrospective study that focused on the characteristics and length of real-time reverse transcription polymerase chain reaction (RT-PCR) positivity and associated hospitalization of confirmed asymptomatic cases of SARS-CoV-2 in the northeast region of Chongqing, China, in the Chongqing Three Gorges Center Hospital and Wanzhou District Hospital since January 2020.

Active contact tracing in China includes nasopharyngeal swab diagnostic testing of all contacts regardless of symptoms. In accordance with Chinese guidelines for the investigation and management of close contacts of COVID-19 cases, close contacts were defined as people who had not taken effective protection while in close contact with suspected or confirmed cases 2 days prior to symptom onset or, if the case was asymptomatic, 2 days before sampling [25]. We screened close contacts from January 2020 through March 2020 in northeast Chongqing by RT-PCR of nasopharyngeal swabs. Only patients with an RT-PCR-confirmed infection and without any apparent symptoms, including (but not limited to) cough, fever, shortness of breathless, and muscle soreness, were enrolled in the study.

These individuals were identified through close symptomatic contacts in both the clinic and the community. In accordance with China's prevention and control policy, all RT-PCR-positive cases were hospitalized and treated, whether they had symptoms or not. We monitored asymptomatic individuals daily during hospitalization to document the development of any signs and symptoms. We recategorized patients who were asymptomatic at baseline to symptomatic if they reported any symptoms. Case definitions of confirmed human infection with SARS-CoV-2 are in accordance with the interim guidance from the World Health Organization. Following a positive SARS-CoV-2 nucleic acid test, a chest CT scan was done [26]. Individuals were then divided into 2 groups based on chest CT scan: a group with changes visible on the radiographic imaging, called asymptomatic COVID-19 patients, and a group without any detectable imaging changes, called asymptomatic with normal chest CT. During hospitalization, all participants, regardless of group, had follow-up chest CT scans every 4 to 5 days until discharge. Individuals initially allocated to the asymptomatic with normal chest CT group were immediately reallocated to the asymptomatic COVID-19 patient group if a new abnormal finding was detected on any chest CT during hospitalization.

With the approval of the ethics committee, we collected both epidemiological data and medical reports for these 2 groups. Epidemiological data collection was achieved by interviewing each patient and their family members, including the dates and times of close contact with (working, living, or gathering together) or to exposure individuals from the affected area (not only Wuhan) with confirmed or suspected SARS-CoV-2 infection. All the data were checked by 2 researchers. If anything was ambiguous, we consulted the attending physician as soon as possible.

\section{Laboratory Tests}

Diagnosis was made by nasopharyngeal swab and RT-PCR to confirm SARS-CoV-2 infection. The virus detection protocol was repeated on subsequent days until a negative nucleic acid test was obtained on 2 consecutive occasions. Other laboratory tests were conducted at admission.

\section{Treatment}

Per standard Chinese protocol, all patients were treated with putative antiviral agents on admission [25]. Fiftyeight of the 63 in the asymptomatic COVID-19 cohort received antiviral treatment with $\alpha$-interferon inhalation (5 million units twice daily) and lopinavir/ritonavir oral (400 mg/100 mg twice daily) with $10 \mathrm{mg}$ thymosin injection every day to improve immunity. Of the remaining 5 patients, 1 received lopinavir/ritonavir (400 mg/100 mg twice daily), Arbidol (200 mg 3 times daily), a-interferon inhalation ( 5 million units twice daily), thymosin, and ribavirin (500 mg twice daily; Figure 1). RT-PCR testing was repeated 5 to 7 days after treatment. Patients were discharged after receiving 2 negative results at least 24 hours apart and a normal chest CT scan [24].

\section{Statistical Analyses}

Continuous variables were expressed as the means and standard deviations or medians with interquartile ranges. Categorical variables were summarized as the counts and percentages in each category. The $t$ test or Mann-Whitney $U$ test was applied 


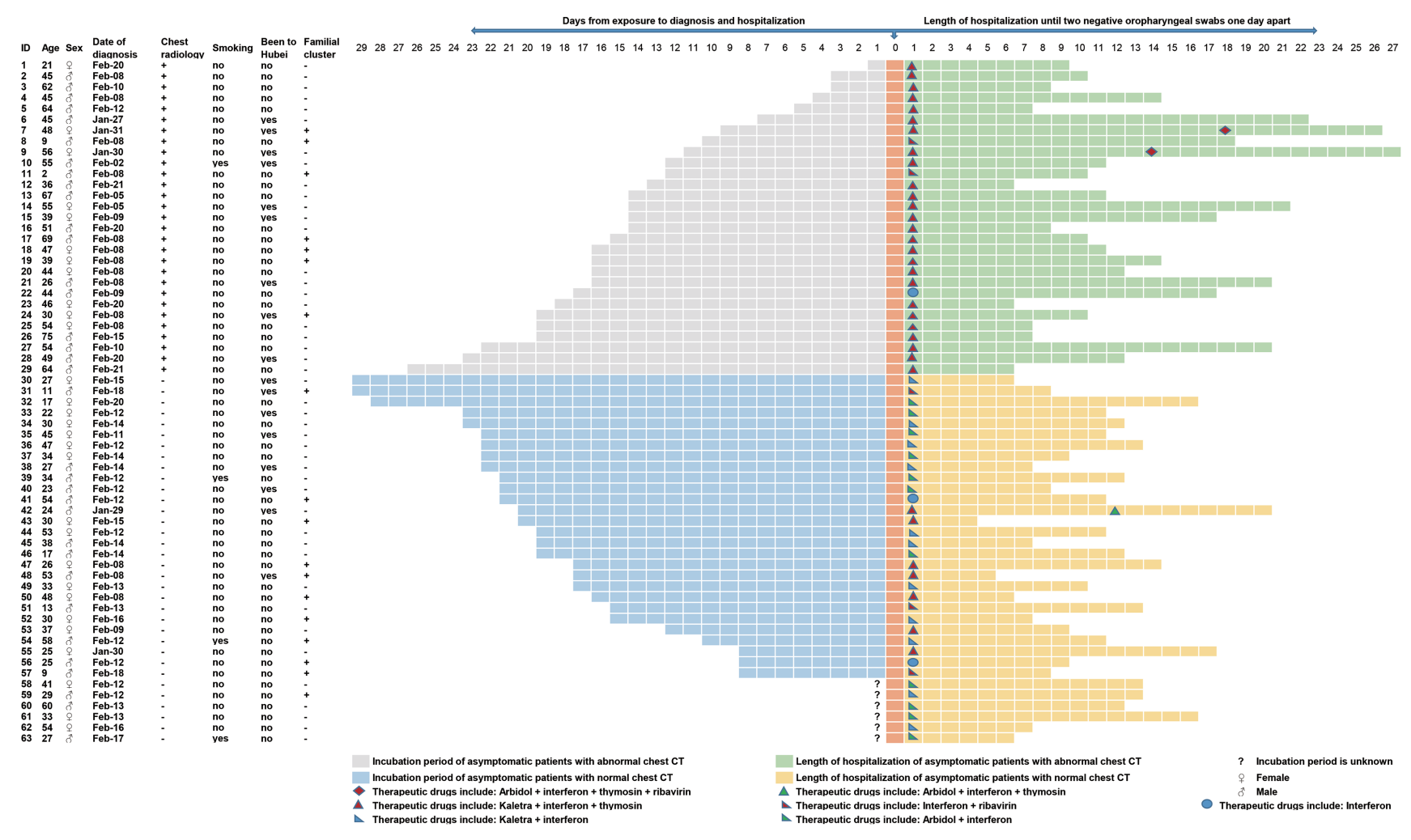

Figure 1. Clinical course and treatment of the 63 asymptomatic patients with severe acute respiratory syndrome coronavirus 2. Abbreviation: CT, computed tomography.

to continuous variables, and the Fisher exact test or Pearson $\chi^{2}$ test was used for categorical variables. All analyses were conducted with SPSS 26.0 .

\section{RESULTS}

\section{General Condition}

Using active contact tracing of index cases with RT-PCR screening, 279 cases were diagnosed with SARS-CoV-2 infection. Among them, 63 had no symptoms either at the time of diagnosis or throughout hospitalization. However, 29 of the 63 cases had abnormal chest CT findings, while the remaining 34 had normal chest CT findings. In total, the mean age was 39.3 years (standard deviation [SD], 16.5). The mean age of cases with abnormal chest CT scans was 46.3 years (SD, 16.8) compared with 33.4 years (SD, $13.8)$ of cases with normal chest CT scans $(P<.05)$. Overall, more than half of the 63 cases were male $(34,54 \%)$; however, $62.1 \%$ of patients with abnormal CT findings were male. Comorbid conditions were present in only 8/63 cases (12.7\%); 6 (20.7\%) in asymptomatic COVID-19 patients and $2(6.1 \%)$ in asymptomatic cases with normal chest CT (Table 1). All of these comorbidities were reported to be very mild by the patients' clinicians.

Regarding potential sources of infection, less than one third ( $\mathrm{n}=17,27 \%$ ) of cases had made short trips to Hubei Province and none had been to the Huanan seafood market. Six cases in the asymptomatic with normal chest CT group could not provide the exact date of close contact with someone with confirmed or suspected SARS-CoV-2 infection. Of the remaining 57 cases, the mean time from exposure to illness onset was 16.0 days (SD, 6.6). In asymptomatic COVID-19 patients, this period was 13.4 days (SD, 6.3), while in asymptomatic cases with normal chest CT scans, it was 18.7 days $(\mathrm{SD}, 5.8)(P<.05)$. Among the 63 cases, there were 18 cases of familial clustering in which 9 cases (14.3\%) transmitted the virus to others outside of their families, 4 from the asymptomatic COVID-19 patients and 5 from the asymptomatic cases with normal chest CT scans (Figure 1).

\section{Clinical Laboratory and Immunologic Findings}

On admission, only 4 of the $63(6.3 \%)$ cases showed leucopenia (white blood cell count $<4 \times 10^{9} / \mathrm{L}$ ) and 4 (6.3\%) showed leukocytosis (white blood cell count $>10 \times 10^{9} / \mathrm{L}$ ). All the cases had a neutrophil count within the normal range. Eight (12.7\%) cases had a low lymphocyte count $\left(<1.0 \times 10^{9} / \mathrm{L}\right)$, including $6(20.7 \%)$ asymptomatic COVID-19 patients and 2 (5.9\%) asymptomatic with normal chest CT scans. Thirty-five of the 63 cases were given procalcitonin tests, with a mean of $0.029 \mathrm{ng} / \mathrm{mL}$ (range, 0.02-0.039), indicating a level within the normal range. Almost half of the 63 cases were tested for cytokines such as interleukin (IL)-6, IL-10, and IL-17 and liver and kidney function, with all showing normal results. In all of these laboratory tests, the $2 \mathrm{im}$ aging groups showed no significant difference (Table 2). 


\begin{tabular}{|c|c|c|c|c|}
\hline \multirow[b]{2}{*}{ Characteristic } & \multirow[b]{2}{*}{ All Asymptomatic Patients ( $N=63$ ) } & \multicolumn{3}{|c|}{ Chest Radiology } \\
\hline & & Normal Chest CT $(n=34)$ & Abnormal Chest CT $(n=29)$ & PValue \\
\hline Age (y) & $39.30 \pm 16.45$ & $33.36 \pm 13.81$ & $46.26 \pm 16.79$ & .001 \\
\hline Age groups (y) & & & & .003 \\
\hline$\leq 18$ & $7(11.1 \%)$ & $5(14.7 \%)$ & $2(6.9 \%)$ & \\
\hline $19-40$ & $25(39.7 \%)$ & $19(55.9 \%)$ & $6(20.7 \%)$ & \\
\hline $41-65$ & $28(44.4 \%)$ & $10(29.4 \%)$ & $18(62.1 \%)$ & \\
\hline$\geq 66$ & $3(4.8 \%)$ & 0 & $3(10.3 \%)$ & \\
\hline Sex & & & & .233 \\
\hline Male & $34(54 \%)$ & $16(47.1 \%)$ & $18(62.1 \%)$ & \\
\hline Female & $29(46 \%)$ & $18(52.9 \%)$ & $11(37.9 \%)$ & \\
\hline Coexisting conditions & & & & .103 \\
\hline Any & $53(84.1 \%)$ & $31(91.2 \%)$ & $22(75.9 \%)$ & \\
\hline Hypertension & $3(4.8 \%)$ & 0 & $3(10.3 \%)$ & \\
\hline Cardiovascular disease & $1(1.6 \%)$ & 0 & $1(3.4 \%)$ & \\
\hline Diabetes & $1(1.6 \%)$ & 0 & $1(3.4 \%)$ & \\
\hline Chronic obstructive pulmonary disease & $1(1.6 \%)$ & $1(2.9 \%)$ & 0 & \\
\hline Systemic lupus erythematosus & $1(1.6 \%)$ & 0 & $1(3.4 \%)$ & \\
\hline Gout & $1(1.6 \%)$ & $1(2.9 \%)$ & 0 & \\
\hline Chronic gastritis & $2(3.2 \%)$ & $1(2.9 \%)$ & $1(3.4 \%)$ & \\
\hline Exposure history in Hubei & & & & .504 \\
\hline Yes & $17(27 \%)$ & $8(23.5 \%)$ & $9(31 \%)$ & \\
\hline No & $46(73 \%)$ & $26(76.5 \%)$ & $20(69 \%)$ & \\
\hline Familial cluster & $18(28.6 \%)$ & $11(32.4 \%)$ & $7(24.1 \%)$ & .472 \\
\hline Days from exposure to diagnosis & $15.98 \pm 6.55(n=57)$ & $18.68 \pm 5.79(n=28)$ & $13.38 \pm 6.25(n=29)$ & .002 \\
\hline Number of others infected & & & & 1.000 \\
\hline No & $54(85.7 \%)$ & $29(85.3 \%)$ & $25(86.2 \%)$ & \\
\hline Yes & $9(14.3 \%)$ & $5(14.7 \%)$ & $4(13.8 \%)$ & \\
\hline
\end{tabular}

Abbreviation: CT, computed tomography.

\section{Clinical Outcome}

Fitness for discharge was based on 2 consecutive negative RT-PCR tests of oropharyngeal swabs and a normal chest CT scan. All patients were discharged, and no one died. The median time between the initial positive RT-PCR test and discharge was significantly longer in asymptomatic COVID-19 patients than in asymptomatic patients with normal chest CT scans, 13.0 and 10.4 days, respectively $(P<.05$; Figure 2$)$.

\section{DISCUSSION}

The number of SARS-CoV-2 infections is still rising rapidly in many parts of the world, and asymptomatic infections likely play a large role in transmission [22]. To our surprise, $23 \%$ of those identified with SARS-CoV-2 infection, including those with abnormal chest CT scans on admission, were completely asymptomatic throughout their infections-a much higher proportion than previously reported [18]. The high proportion may be related to the extensive and strict close-contact screening policy adopted locally that resulted in early detection and treatment of cases. This finding suggests that there are actually more asymptomatic COVID-19 cases than previously thought, highlighting asymptomatic cases as a noteworthy source of infection. A recent study reported that the viral load in asymptomatic patients is similar to that in symptomatic patients, further underscoring the transmission potential of these asymptomatic patients [27]. Together, these results suggest that strict isolation and screening should be carried out in all asymptomatic close contacts. CT scans can assist in the detection of asymptomatic pneumonia but cannot identify asymptomatic cases with normal chest CT scans. If nucleic acid testing were reserved only for suspected patients with obvious symptoms, many contacts with mild symptoms or those who are asymptomatic would be missed, as would their contacts.

Since there are no obvious symptoms, asymptomatic cases typically remain undiagnosed for a relatively long time. In our study, the mean latency between close contact and diagnosis was 16.0 days, with a maximum of 29 days. This suggests that RT-PCR screening should be undertaken even if an individual shows no symptoms more than 14 days after close contact with an infected person. In asymptomatic patients, those whose CT scans show signs of pneumonia are typically diagnosed earlier than those without such signs.

Because the pathogenesis of COVID-19 is not well understood, all nucleic acid-positive cases received antiviral treatment to prevent progression of the disease. Although the 


\begin{tabular}{|c|c|c|c|c|}
\hline \multirow[b]{2}{*}{ Variable } & \multirow[b]{2}{*}{ All Asymptomatic Patients ( $N=63$ ) } & \multicolumn{3}{|c|}{ Chest Radiology } \\
\hline & & Normal Chest CT $(n=34)$ & Abnormal Chest CT $(n=29)$ & $P$ Value \\
\hline White blood cell count $\left(\times 10^{9} / \mathrm{L}\right)$ & $6.81 \pm 1.98$ & $7.10 \pm 2.04$ & $6.47 \pm 1.88$ & .207 \\
\hline White blood cell count $\left(\times 10^{9} / \mathrm{L}\right)$ & & & & .854 \\
\hline$<4$ & $4(6.3 \%)$ & $2(5.9 \%)$ & $2(6.9 \%)$ & \\
\hline $4-10$ & $55(87.3 \%)$ & $29(85.3 \%)$ & $26(89.7 \%)$ & \\
\hline$>10$ & $4(6.3 \%)$ & $3(8.8 \%)$ & $1(3.4 \%)$ & \\
\hline Neutrophil count $\left(\times 10^{9} / \mathrm{L}\right)$ & $4.59 \pm 1.78$ & $4.91 \pm 1.89$ & $4.21 \pm 1.59$ & .122 \\
\hline Lymphocyte count $\left(\times 10^{9} / \mathrm{L}\right)$ & $1.61(1.24-1.92)$ & $1.66(1.35-1.95)$ & $1.59(1.19-1.93)$ & .490 \\
\hline Lymphocyte count $\left(\times 10^{9} / \mathrm{L}\right)$ & & & & .129 \\
\hline$<1.0$ & $8(12.7 \%)$ & $2(5.9 \%)$ & $6(20.7 \%)$ & \\
\hline$\geq 1.0$ & $55(87.3 \%)$ & $32(94.1 \%)$ & $23(79.3 \%)$ & \\
\hline CD4+ T lymphocytes (cells/uL) & $566(371-762)(n=36)$ & $653(460-857)(n=8)$ & $549(332-732)(n=28)$ & .313 \\
\hline CD8+ T lymphocytes (cells/ul) & $322(252-501)(n=36)$ & $410(277-614)(n=8)$ & $317(229-489)(n=28)$ & .304 \\
\hline Procalcitonin (ng/mL) & $0.029(0.02-0.039)(n=35)$ & $0.036(0.024-0.045)(n=8)$ & $0.028(0.02-0.039)(n=27)$ & .312 \\
\hline Albumin & $43.2(41.4-45.4)(n=36)$ & $43(40.4-45.4)(n=9)$ & $43.2(41.4-45.4)(n=27)$ & .534 \\
\hline Alanine aminotransferase (U/L) & $16.8(12.7-28.2)(n=36)$ & $15.1(9.9-22.3)(n=9)$ & $17.4(13.2-30.4)(n=27)$ & .250 \\
\hline Aspartate aminotransferase (U/L) & $18.8(14.8-22.6)(n=36)$ & $19.3(13-22.5)(n=9)$ & $18.7(15.1-23)(n=27)$ & .511 \\
\hline Blood urea nitrogen $(\mathrm{mmol} / \mathrm{L})$ & $4.5(3.7-5.2)(n=36)$ & $4.5(3.7-4.9)(n=9)$ & $4.4(3.7-5.5)(n=27)$ & .728 \\
\hline Creatine $(\mu \mathrm{mol} / \mathrm{L})$ & $64(53-77)(n=36)$ & $54(46-79)(n=9)$ & $65(55-75)(n=27)$ & .454 \\
\hline IL-6 (pg/mL) & $3.71(1.94-5.46)(n=33)$ & $4.97(3.19-6.55)(n=6)$ & $2.72(1.76-4.48)(n=27)$ & .430 \\
\hline IL-10 (pg/mL) & $2.62(2.34-3.14)(n=33)$ & $2.68(2.57-2.97)(n=6)$ & $2.56(2.25-3.19)(n=27)$ & .484 \\
\hline IL-17 (pg/mL) & $1.38(1.18-1.63)(n=33)$ & $1.37(1.31-1.49)(n=6)$ & $1.43(1.13-1.69)(n=27)$ & .981 \\
\hline
\end{tabular}

Abbreviation: CT, computed tomography; IL, interleukin.

efficacy of antiviral therapy is still unknown, it is possible that treatment prevented the progression of disease in this cohort. Asymptomatic COVID-19 patients with abnormal chest CT scans showed improvement over time, suggesting that these patients may have benefitted from the antiviral therapy. Even with antiviral therapy, the average time for viral nucleic acid testing assays to return to negative in asymptomatic COVID-19 patients after treatment was 13 days, which is not shorter than

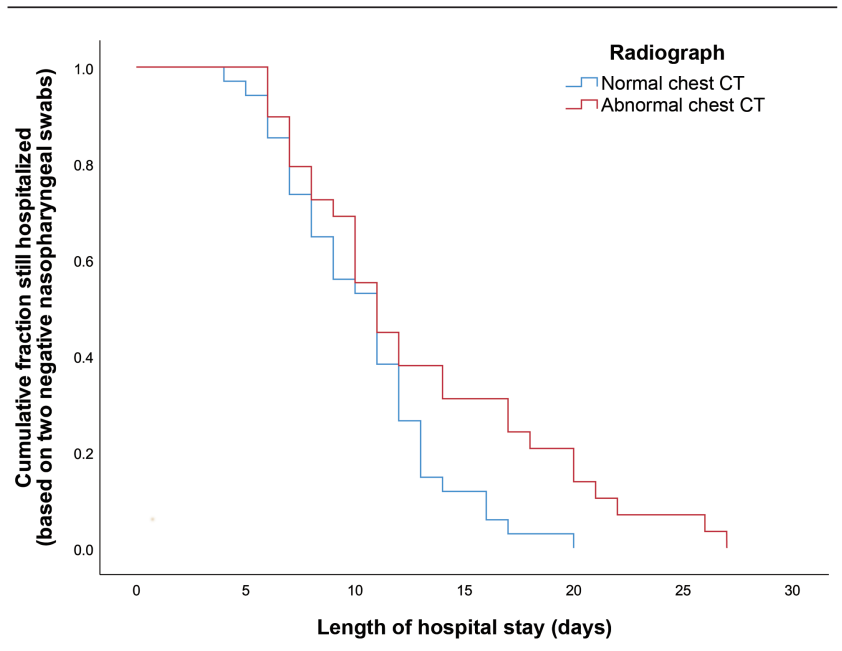

Figure 2. Outcomes and mean and median for length of hospital stay of the 63 asymptomatic patients with severe acute respiratory syndrome coronavirus 2. Abbreviation: CT, computed tomography. the time reported for mild symptomatic patients [12]. However, cases with abnormal chest CT scans took significantly longer to become RT-PCR-negative than those without chest CT abnormalities. These characteristics indicate that asymptomatic COVID-19 cases are important recessive sources of infection. Thus, diagnosing all SARS-CoV-2 infections cases, including those that are very mild, subclinical, or asymptomatic, as soon as possible and immediately isolating them is likely to be critical to cutting off the source of infection. Although they have no symptoms or even no abnormal chest images, they spread the virus, causing infection and morbidity [20].

We found that 18 of the 63 asymptomatic cases (28.6\%) had infection associated with familial clustering, indicating that asymptomatic infections can be identified through screening family members of an index case. The high proportion may be related to the extent and length of close contact, as well as the relative ease of tracking and screening family members $[8,9]$. However, it is sometimes difficult to tell who transmitted the virus, the asymptomatic case or the infected family member [8]. It also suggests that occult transmission of SARS-CoV-2 may exist. Only $14 \%$ of the asymptomatic cohort infected others, occurring equally between those with and without chest CT abnormalities. However, it is certain that there are still a few asymptomatic cases that can cause transmission [28]. Serum antibody tests may provide a lowcost and rapid method for screening and could come to play an important role in the auxiliary diagnosis of SARS-CoV-2 
infection [29]. As a group, the asymptomatic cases in our study were younger and had fewer comorbidities compared with severe cases $[11,12]$. Research shows that the adaptive immune response against the virus in people with asymptomatic infection is stronger than in those with symptomatic infection [30, 31]. Asymptomatic individuals without imaging abnormalities are younger than those with pneumonia. From our results, it seems that young individuals with normal immune function and without comorbidities are more likely to become asymptomatically infected.

All patients in the study, with and without abnormal chest CT scans, steadily improved and were discharged smoothly after testing negative by RT-PCR. However, we do not know whether these asymptomatic cases, particularly those who had developed an abnormal chest CT scan, would have progressed to more severe disease without early antiviral treatment. The results also suggest the need for rigorous randomized, controlled studies to determine what antiviral therapies are safe and effective and whether early antiviral treatment for asymptomatic RT-PCR+ individuals can prevent progression of disease and/or the length of time cases remain RT-PCR+. Finally, SARS-CoV-2 vaccine study designs should not simply use symptomatic COVID-19 as an end point but should include asymptomatic infections as well.

This study has several limitations. First, only 63 asymptomatic cases were included; as such it may be that we found no other associated epidemiological factors due to sample size. Second, we used a qualitative RT-PCR test and could not quantitate viral load; thus, asymptomatic cases with low viral load may have been missed. Third, a precise incubation period and transmissibility are difficult to document due to the concealed nature of asymptomatic infection. Finally, our study does not provide a mechanistic explanation for asymptomatic status. A study that links asymptomatic status with differences in individual immunity, virus serotypes, viral load or other factors would contribute useful insights.

In conclusion, we provide an initial assessment of epidemiological characteristics of asymptomatic infections of SARS-CoV-2. Early identification of SARS-CoV-2 cases with subsequent isolation and treatment may contribute to decreased transmission and mortality.

\section{Notes}

Author contributions. Y. H. and J. IX. conceived of and designed the study, had full access to all data, and take responsibility for the integrity of the data and the accuracy of the data analysis. Y. W., J. T., Y. Q., T. X., J. hX., Y. C., J. rL., and J. IX. contributed to data acquisition. Y. H., Y. W., E. S. H., and J. T. contributed to writing the report. J. hL. contributed to the statistical analysis. All authors contributed to data analysis or data interpretation and reviewed and approved the final version.

Acknowledgments. The authors thank Dr Saffron Willis-Owen and Dr Youming Zhang from the National Heart and Lung Institute, Imperial College London, and the Dr Robert Taylor from the National Institute of Allergy and Infectious Diseases, National Institutes of Health, for critical reading and editing of the manuscript. With the permission of the corresponding authors, we can provide participant data without names and identifiers. Data can be provided after the article is published. Once the data is made public, the research team will provide an email address for communication. The corresponding authors have the right to decide whether to share the data or not based on the research objectives and plan provided. This study was reviewed and approved by the Medical Ethical Committee of Daping Hospital and Chongqing Three Gorges Central Hospital.

Financial support. This study was supported by Excellent Talent Pool grants for Y. W. and Y. H. and Training Plan of Clinical Medical Scientific Research Talents for Y. H. from the Army medical university (2018XLC1015).

Potential conflicts of interest. The authors: No reported conflicts of interest. All authors have submitted the ICMJE Form for Disclosure of Potential Conflicts of Interest.

\section{References}

1. World Health Organization. Novel coronavirus (COVID-19) situation. 2020. Available at: https://experience.arcgis.com/experience/685d0ace521648f8a5beee ee1b9125cd. Accessed 21 February 2020.

2. Lipsitch M, Cohen T, Cooper B, et al. Transmission dynamics and control of severe acute respiratory syndrome. Science $2003 ; 300: 1966-70$.

3. Wallinga J, Teunis P. Different epidemic curves for severe acute respiratory syndrome reveal similar impacts of control measures. Am J Epidemiol 2004; 160:509-16.

4. Lin Q, Chiu AP, Zhao S, He D. Modeling the spread of Middle East respiratory syndrome coronavirus in Saudi Arabia. Stat Methods Med Res 2018; 27:1968-78.

5. Wang C, Horby PW, Hayden FG, Gao GF. A novel coronavirus outbreak of global health concern. Lancet 2020; 395:470-3.

6. van Doremalen N, Bushmaker T, Morris DH, et al. Aerosol and surface stability of SARS-CoV-2 as compared with SARS-CoV-1. N Engl J Med 2020; 382:1564-7.

7. New coronavirus pneumonia prevention and control program. 5 th ed. (in Chinese). 2020. Available at: http://www.nhc.gov.cn/xcs/zhengcwj/202002/a 5d6f7b8c48c451c87dba14889b30147.shtml. Accessed 21 February 2020.

8. Pan X, Chen D, Xia Y, et al. Asymptomatic cases in a family cluster with SARSCoV-2 infection. Lancet Infect Dis 2020; doi: S1473-3099(20)30114-6

9. Yu P, Zhu J, Zhang Z, et al. A familial cluster of infection associated with the 2019 novel coronavirus indicating potential person-to-person transmission during the incubation period. J Infect Dis 2020. doi: 10.1093/infdis/jiaa077

10. Li Q, Guan X, Wu P, et al. Early transmission dynamics in Wuhan, China, of novel coronavirus-infected pneumonia. N Engl J Med 2020; 382:1199-207.

11. Huang C, Wang Y, Li X, et al. Clinical features of patients infected with 2019 novel coronavirus in Wuhan, China. Lancet 2020; 395:497-506.

12. Chen N, Zhou M, Dong X, et al. Epidemiological and clinical characteristics of 99 cases of 2019 novel coronavirus pneumonia in Wuhan, China: a descriptive study. Lancet 2020; 395:507-13.

13. Chan JF, Yuan S, Kok KH, et al. A familial cluster of pneumonia associated with the 2019 novel coronavirus indicating person-to-person transmission: a study of a family cluster. Lancet 2020; 395:514-23.

14. Li JY, You Z, Wang Q, et al. The epidemic of 2019-novel-coronavirus (2019$\mathrm{nCoV})$ pneumonia and insights for emerging infectious diseases in the future. Microbes Infect 2020; doi: S1286-4579(20)30030-7

15. Lai CC, Shih TP, Ko WC, et al. Severe acute respiratory syndrome coronavirus 2 (SARS-CoV-2) and coronavirus disease-2019 (COVID-19): the epidemic and the challenges. Int J Antimicrob Agents 2020; 55:105924.

16. Zhu N, Zhang D, Wang W, et al. A novel coronavirus from patients with pneumonia in China, 2019. N Engl J Med 2020; 382:727-33.

17. Zhou P, Yang XL, Wang XG, et al. A pneumonia outbreak associated with a new coronavirus of probable bat origin. Nature 2020; 579:270-3.

18. The Novel Coronavirus Pneumonia Emergency Response Epidemiology Team. The epidemiological characteristics of an outbreak of 2019 novel coronavirus diseases (COVID-19) in China. Zhonghua Liu Xing Bing Xue Za Zhi 2020; 41:145-51.

19. Hu Z, Song C, Xu C, et al. Clinical characteristics of 24 asymptomatic infections with COVID-19 screened among close contacts in Nanjing, China. Sci China Life Sci 2020; 63:706-11.

20. Bai Y, Yao L, Wei T, et al. Presumed asymptomatic carrier transmission of COVID-19. JAMA 2020. doi: 10.1001/jama.2020.2565

21. Qiu J. Covert coronavirus infections could be seeding new outbreaks. Nature 2020. doi: $10.1038 / \mathrm{d} 41586-020-00822-\mathrm{x}$ 
22. Al-Tawfiq JA. Asymptomatic coronavirus infection: MERS-CoV and SARS-CoV-2 (COVID-19). Travel Med Infect Dis 2020; 101608. doi: 10.1016/j.tmaid.2020.101608

23. Gao WJ, Li LM. Advances on presymptomatic or asymptomatic carrier transmission of COVID-19. Zhonghua Liu Xing Bing Xue Za Zhi 2020; 41:485-8.

24. Wang Y, Wang Y, Chen Y, et al. Unique epidemiological and clinical features of the emerging 2019 novel coronavirus pneumonia (COVID-19) implicate special control measures. J Med Virol 2020. doi: 10.1002/jmv.25748

25. Chinese Center For Disease Control and Prevention. The guideline for the investigation and management of close contacts of COVID cases. 2020. Available at: http://www.gov.cn/xinwen/2020-01/26/content_5472235.htm.

26. Office of the National Health Commission. The guideline on diagnosis and treatment of COVID (The Trial, fourth edition). 2020. Available at: http://swjj.gzlps. gov.cn/gzdt/gzdt_45694/202002/t20200221_50515109.html.
27. Zou L, Ruan F, Huang M, et al. SARS-CoV-2 viral load in upper respiratory specimens of infected patients. N Engl J Med 2020; 382:1177-9.

28. MO X, Qin W, Qihua F, Ming G. Understanding the influence factors in viral nucleic acid test of 2019 novel coronavirus (2019-nCoV). Chin J Lab Med 2020 43:E002.

29. Zhang W, Du RH, Li B, et al. Molecular and serological investigation of 2019$\mathrm{nCoV}$ infected patients: implication of multiple shedding routes. Emerg Microbes Infect 2020; 9:386-9.

30. Simon-Loriere E, Duong V, Tawfik A, et al. Increased adaptive immune responses and proper feedback regulation protect against clinical dengue. Sci Transl Med 2017; 9. pii: eaal5088.

31. Guan WJ, Ni ZY, Hu Y, et al. Clinical characteristics of coronavirus disease 2019 in China. N Engl J Med 2020. doi: 10.1056/NEJMoa2002032 